\title{
Proximal ulna stress fracture and stress reaction of the proximal radius associated with the use of crutches: a case report and literature review
}

\author{
Nanda Venkatanarasimha $\cdot$ Sridhar Kamath $\cdot$ \\ G. Kambouroglou $\cdot$ Simon J. Ostlere
}

Received: 13 January 2009/Accepted: 7 July 2009/Published online: 26 August 2009

(C) Springer-Verlag 2009

\begin{abstract}
We report a case of complete stress fracture of the ulna and stress reaction of the radius resulting from the use of crutches in an overweight patient with severe lower extremity arthritis. Plain radiograph showed an undisplaced complete fracture of the proximal metaphysis of the ulna. Magnetic resonance imaging (MRI) was performed to exclude a pathological cause in view of the unusual fracture site, which confirmed the plain radiographic findings and additionally demonstrated a stress reaction in the proximal radius. There are three cases of stress fracture of the ulnar diaphysis resulting from the use of crutches reported previously in the English literature and a further case of bilaterally symmetrical ulnar diaphysial fracture reported in the Danish literature. We report the first case of ulnar metaphysis stress fracture with concomitant stress reaction of the radius.
\end{abstract}

Keywords Stress fracture $\cdot$ Ulna $\cdot$ Radius .

Magnetic resonance imaging

N. Venkatanarasimha

Department of Radiology, Peninsula Radiology Academy, Derriford Hospital, Plymouth International Business Park, Derriford Road, Plymouth PL6 5WR, UK

e-mail: nandashettykv@yahoo.com

S. Kamath $(\varangle) \cdot$ S. J. Ostlere

Department of Radiology, Nuffield Orthopaedic Centre,

Windmill Road, Headington, Oxford OX3 7LD, UK

e-mail: kamathsridhar@yahoo.com

S. J. Ostlere

e-mail: Simon.Ostlere@NOC.ANGLOX.NHS.UK

G. Kambouroglou

Oxford Trauma Unit, John Radcliffe Hospital,

Headington, Oxford, UK

\section{Introduction}

Stress fractures of the ulna and radius are rare. The middle third of the ulna is the commonest site as this region has the thinnest cortex and smallest crosssectional area morphologically compared to the proximal or the distal third, and is vulnerable to stress from torsional forces [1]. Stress fractures of the ulna have been reported to be associated with certain sports such as tennis. We present a case of a spontaneous complete fracture of the ulna metaphysis with concomitant stress reaction of the proximal radius occurring in a patient who had been dependent on below elbow crutches for 15 months. The case acts as a reminder that spontaneous complete fractures may be the end result of abnormal stress, and that stress fractures of the ulna may be related to the use of crutches.

\section{Case report}

A 60-year-old left hand-dominant woman presented 3 days after hearing a crack in the left forearm whilst taking her jumper off. She had subsequently noticed pain and swelling at this site. There was no history of preceding trauma. Relevant past medical history included severe osteoarthritis of the hips, knees and back requiring below elbow crutches to mobilise. She was taking non-steroidal anti-inflammatory medications for pain relief. On examination, there was diffuse swelling over the proximal ulna associated with tenderness over the site. Plain radiographs (Fig. 1) showed an undisplaced transverse fracture through the proximal metaphysis of the ulna. She was treated conservatively and provided with alternative crutches to avoid weight bearing on the forearm. 


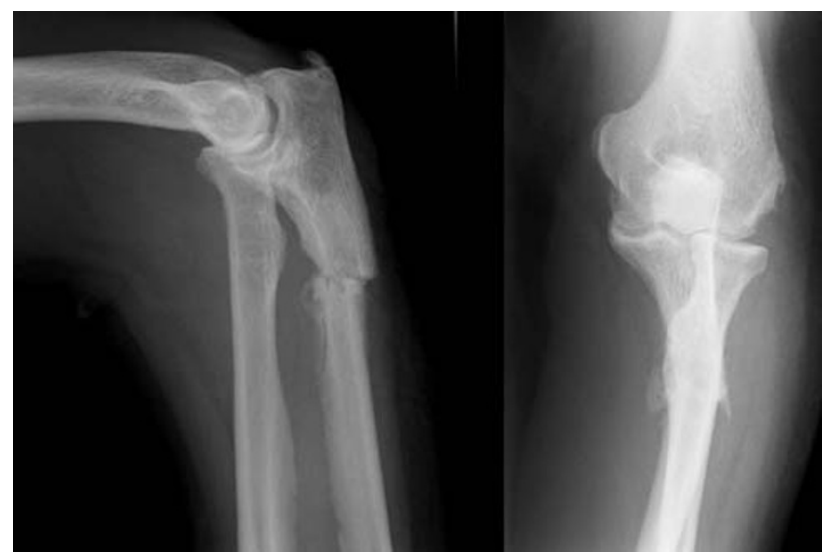

Fig. 1 Anterior-posterior (AP) and lateral plain radiographs demonstrate a transverse fracture through the proximal metaphysis of the ulna

A repeat plain radiograph at 5 weeks showed signs of fracture union. However, in view of the unusual site of the fracture, a magnetic resonance imaging (MRI) scan was requested. MRI was performed on a 1.5 tesla Siemens scanner using a body coil as the patient's body habitus did not permit the use of a surface coil available in our institution. The quality of the resulting scan was therefore slightly suboptimal. MRI showed features of a healing stress fracture in the proximal ulna. In addition, there was bone marrow oedema in the proximal radius without a definite fracture line-appearances consistent with a stress reaction (Fig. 2a-c). There was no evidence of neoplasm or infection. A subsequent computed tomography (CT) scan showed no fracture line in the radius and no signs of underlying bone pathology in either of the bones (Fig. 3). The patient reported improvement in symptoms upon clinical review. We have received written consent from the patient for publication of this article.

\section{Discussion}

Stress fracture of the ulna due to the use of crutches is rare. Previously published case reports describe stress fracture/ reaction of the ulna diaphysis [2-5], of which two patients had complete fractures. We report a case of a complete stress fracture of the ulnar metaphysis resulting from the use of crutches. The association of concomitant stress reaction of the radius has not been reported previously.

Troell et al. [6] first described stress fracture of the ulna in 1941. Since this initial observation, ulnar stress fractures have been described in a variety of sporting activities including tennis [7], weight lifting [8], baseball [9], polo [10] and Japanese fencing [11].

Stress fractures occurring in the non-weight bearing bones can cause diagnostic confusion with pathological
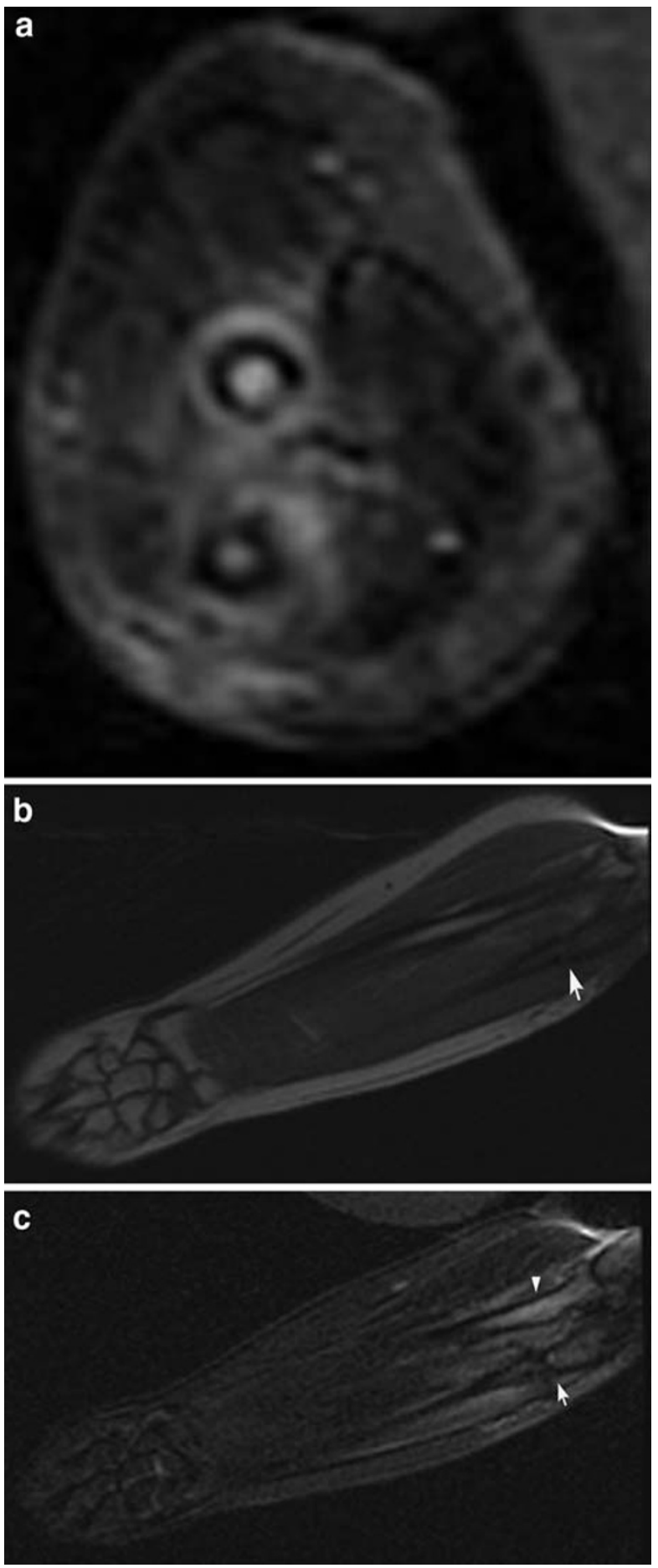

Fig. 2 Magnetic resonance imaging (MRI) of the left forearm. a Axial short tau inversion recovery (STIR) demonstrates fracture of the proximal ulna and surrounding soft tissue oedema. Marrow oedema within the proximal radius is also seen. b Coronal T1W image demonstrates the transverse fracture of the proximal ulnar metaphysis (arrow) and low signal changes in the proximal radius at the same level as the ulnar fracture. c Coronal STIR demonstrates the ulnar fracture (arrow) High signal changes in the proximal radius (arrow head) representing bone marrow oedema again demonstrated. No sinister features are seen 




Fig. 3 Computed tomography (CT) scan of the left forearm; Sagittal reformat shows the transverse fracture through the proximal ulnar metaphysis with associated callous formation

fractures if the clinical history is not clear or when such fractures occur in the setting of an underlying primary malignancy elsewhere.

The first report of a stress fracture of the ulna resulting from the use of crutches was described by McGoldrick and O'Brien [3], in a patient who was non-weight bearing following treatment for osteochondritis dissecans of the knee. They described bilateral complete stress fractures involving the mid-diaphysis of the ulna occurring at identical sites, and postulated direct contact of the ulna with the upper crutch ring in combination with a forward bending force as the likely cause. A similar case of a bilaterally symmetrical complete stress fracture of the ulnar diaphysis, which resulted from the use of walking aid for a year, was reported in a 95-year-old patient [5]. Garcia Suarez et al. [4] reported a further case of an incomplete crutch-related stress fracture of the ulna occurring at the junction of the middle and distal third in a patient treated for osteochondritis of the knee. They postulated that cyclical weight bearing on crutches, with the wrist in a position of flexion, ulnar deviation and pronation, increased the axial load through the ulna, leading to fracture. More recently, Amin et al. [12] reported a case of ulnar stress reaction from crutch use in a patient who had undergone above-knee amputation for recurrent osteosarcoma of the tibia. None of the reported cases showed concomitant stress reaction of the ipsilateral radius.

In our case, it was thought that the likely mechanism of injury was due to a combination of tractional and torsional force, together with direct pressure on the proximal forearm bones from the upper crutch ring as postulated in the previous case reports [2-4]. Furthermore, MRI and CT showed no evidence of an underlying lesion to suggest a pathological fracture in our case, and the fracture progressed to union without complication.

In conclusion, both clinicians and radiologists involved in the management of such patients should be aware of stress fracture/reaction of the forearm bones that can occur from the use of crutches, which may help in early detection and treatment, preventing further disability in these patients.

Conflict of interest statement The authors declare that they have no conflict of interest related to the publication of this manuscript.

\section{References}

1. Jones GL (2006) Upper extremity stress fracture. Clin Sports Med 25:159-174

2. Amin A, Singh V, Saifuddin A, Briggs TWR (2004) Ulnar stress reaction from crutch use following amputation for tibial osteosarcoma. Skeletal Radiol 33:541-544

3. McGoldrick F, O'Brien TM (1988) Bilateral stress fractures of the ulna. Injury 19:360-361

4. Gracia Suarez G, Garcia Garcia J, Perez Carro L (2001) Stress fracture of the ulna associated with crutch use. J Orthop Trauma 15:524-525

5. Sorensen L (1992) Bilateral symmetrical stress fracture of the ulna (in Danish). Ugeskr Laeger 154:1850-1851

6. Troell A, Lauritzen G, Moller A (1941) Fractures of apparently healthy bone without unquestionably true element of accident. Acta Chir Scand 84:226

7. Rettig AC (1983) Stress fracture of the ulna in an adolescent tournament tennis player. Am J Sports Med 11:103-106

8. Hamilton HK (1984) Stress fracture of the diaphysis of the ulna in a body builder. Am J Sports Med 12:405-406

9. Hamilton BH, Colsen E, Brukner P (1999) Stress fracture of the ulna in a baseball pitcher. Clin J Sport Med 9:231-232

10. Clark RJ, Sizer PS Jr, Slauterbeck J (2002) Stress Fracture of the ulna in a male competitive polo player. Am J Sports Med 30:130 132

11. Sujino T, Ohe T, Shinozuka M (1998) Bilateral stress fractures of the ulnae in a Kendo (Japanese fencing) player. Br J Sports Med 32:340-342

12. Craig JG, Widman D, van Holsbeeck M (2003) Longitudinal stress fracture: patterns of edema and the importance of the nutrient foramen. Skeletal Radiol 32:22-27 